\title{
A Fuzzy Non-linear Similarity Measure for Case-Based Reasoning Systems for Radiotherapy Treatment Planning
}

\author{
Rupa Jagannathan ${ }^{1}$, Sanja Petrovic ${ }^{1}$, Angela McKenna ${ }^{2}$, and Louise Newton ${ }^{2}$ \\ ${ }^{1}$ Automated Scheduling, Optimisation and Planning Research Group \\ School of Computer Science, University of Nottingham, Nottingham, UK \\ $\{r x j, \operatorname{sxp}\} @ \mathrm{cs}$. nott.ac.uk \\ ${ }^{2}$ Department of Medical Physics \\ Nottingham University Hospitals NHS Trust Nottingham, UK \\ \{angela.mckenna, louise.newton\}@nuh.nhs.uk
}

\begin{abstract}
This paper presents a decision support system for treatment planning in brain cancer radiotherapy. The aim of a radiotherapy treatment plan is to apply radiation in a way that destroys tumour cells but minimizes the damage to healthy tissue and organs at risk. Treatment planning for brain cancer patients is a complex decision-making process that relies heavily on the subjective experience and expert domain knowledge of clinicians. We propose to capture this experience by using case-based reasoning. Central to the working of our case-based reasoning system is a novel similarity measure that takes into account the non-linear effect of the individual case attributes on the similarity measure. The similarity measure employs fuzzy sets. Experiments, which were carried out to evaluate the similarity measure using real brain cancer patient cases show promising results.
\end{abstract}

Keywords: Case-based Reasoning, Fuzzy Logic, Radiotherapy Treatment Planning, Decision-Support Systems.

\section{Introduction}

Radiotherapy is a treatment method that uses ionizing radiation in cancer control. The aim of radiotherapy treatment planning (RTP) is to deliver a tumouricidal radiation dose over the tumour region while minimizing the radiation received by healthy tissue and critical organs in the vicinity of the tumour. To realize this goal, a detailed treatment plan is created for each patient that describes exactly how a patient will be irradiated to achieve the required dose distribution. Parameters of RTP include the number of beams, the gantry angle of beams (the angle by which the apparatus applying the radiation beam is moved around the patient's body) and wedges that shape the beam. Oncologists, together with medical physicists, use their subjective experience and expert clinical knowledge to generate treatment plans. The RTP decision support system, under development in collaboration with the Nottingham University Hospitals, City Hospital campus, uses case-based reasoning to capture this experience and aid oncologists in the computation of plan parameters. 
Case-based reasoning (CBR) is based on the concept that the solution of a problem can be derived from the solutions of similar problems [1]. The main advantage of CBR in RTP over numerical optimisation [2] and rule-based methods [3] is its capability to utilise non-quantifiable or subjective knowledge and benefit from the successes, errors and failures of previous treatment plans. In addition, since treatment plan generation is based on existing plans, new plans can be generated quickly and in line with the institution's preferences. Although, CBR has been widely applied in healthcare systems in the diagnosis and treatment of disease $[4,5]$, the research done on CBR in radiotherapy treatment planning has been limited. Case based reasoning has been used in dose planning for prostate cancer by Song et al [6] and Mishra et al [12]. The CBR system Roentgen, designed by Berger [7], aids radiotherapy planning for thorax cancer and retrieves cases from its case archive based on the similarity between patient geometric descriptors. However, no implementation details or experimental evaluation of the method are furnished. The work presented in this paper builds up on Berger's idea of using the geometry of the patient as input to the CBR system and implements this concept using a fuzzy non-linear similarity measure.

The choice of the similarity measure, which calculates how similar cases in the case base are to the target case, is fundamental. The popular nearest neighbour method [8] matches each attribute in the target case to its corresponding attribute in the archive case. The aggregate similarity is given by the weighted sum of the individual similarities. However, if the similarity values with respect to individual attributes are very different in terms of their distribution or range they can not be directly compared or summed up to give an accurate representation of the aggregate similarity between two cases. Another common assumption of many CBR systems is that the similarity with respect to a single case attribute influences the aggregate similarity linearly over its range. In reality, however, the attribute similarity between two cases often behaves in a non-linear fashion $[9,10]$. That is, a high similarity value with respect to a non-linear attribute indicates a high suitability of the case solution to the target case but also a very low similarity of non-linear attribute has a detrimental effect on the suitability of the case. To overcome these limitations, our CBR system uses a novel fuzzy non-linear similarity measure. The performance of the proposed similarity measure is evaluated using real brain cancer patient cases obtained from the Nottingham City Hospital.

The paper is organized as follows. Section 2 describes the architecture of the CBR system and the non-linear fuzzy similarity measure. Section 3 presents some results obtained by comparing the performance of the standard weighted nearest neighbour and the fuzzy non-linear similarity measure. Section 4 discusses future research directions and concludes our work.

\section{Architecture of the CBR System}

Each case in the developed CBR system contains a patient description and a suitable treatment plan detailing the RTP beam configuration. The patient description consists of spatial information regarding the location of the planning target volume containing the tumour (PTV) and the organs at risk (OAR). The OAR commonly include the spinal cord, the eyes, the lens, the optic nerve and chiasm, the brainstem and the 
pituitary gland. The cases are sorted according to the OAR present in the vicinity of the PTV. Since procuring clinical patient data is a slow and difficult process, the case base currently contains only 24 brain cancer patient cases. However, we are continuously adding more cases to our case base as they become available.

\subsection{Case Attributes}

Case attributes identified to be relevant for similarity between two patients describe the location of the tumour and the spatial relationship between the tumour and the OAR. These attributes determine the geometry of a patient and are computed using $3 \mathrm{D}$ coordinates representing the PTV and OAR structures, which are extracted from the CT DICOM [11] image header files created for each patient. The following geometric descriptors are used.

- Volume of PTV, denoted by $V$.

- Distance between tumour and OAR, denoted by $E$, is defined as the minimum edge-to-edge distance between the tumour and the OAR.

- Angle between tumour and OAR, denoted by $A$, is defined as the angle between the line connecting the origin of the image patient coordinate system and the tumour centroid and the line connecting the origin and the OAR centroid.

\subsection{A Fuzzy Similarity Measure}

A good definition of a similarity measure between two cases is of crucial importance for the retrieval process, which should retrieve from the case base a case with a treatment plan suitable for the target patient. A suitable treatment plan is one whose plan parameters achieve the desired radiation dose distribution in the target case patient. After consultation with medical physicists, we have arrived at the assumption that patients with similar geometric descriptors have similar treatment plans. The CBR system Roentgen is based on this assumption as well [7]. In the retrieval process, the similarity with respect to the geometric descriptors is calculated between the target case and those cases in the case base that contain the same OAR.

The values of the three geometric descriptors are normalized to take values from the interval $[0,1]$. Let $\mathrm{C}_{\mathrm{T}}$ be the target case and $\mathrm{C}_{\mathrm{C}}$ be a case from the case-base. The distance $d_{l}$ between attribute value $v_{T}$ in target case $\mathrm{C}_{\mathrm{T}}$ and attribute value $v_{C}$ in case $\mathrm{C}_{\mathrm{C}}$ with respect to attribute $l$, where $l=A, E$ and $V$, is calculated as follows:

$$
d_{l}=v_{T}-v_{C} .
$$

Then, the similarity between $\mathrm{C}_{\mathrm{T}}$ and $\mathrm{C}_{\mathrm{C}}$ with respect to attribute $l$ is:

$$
s_{l}=1-d_{l} .
$$

The similarity values with respect to each attribute have to be combined into an aggregate similarity value between two cases. In the nearest neighbour method, the aggregate similarity is generally defined as the weighted sum of the individual attribute similarities. This method however does not take into account the variations 
in the distribution of the similarity values of different attributes. In order to obtain an idea of the distribution of attribute similarities, we calculate the similarity between each case and every other case (with the same OAR) in our case base in a leave-one out fashion considering one attribute a time. Figure 1 shows the similarity calculated between all possible pairs of patient cases in the case base (arranged in decreasing order of similarity) for each attribute. The data suggests a trend in the distribution of the attribute similarity values between cases. We can see that the distribution and range of the similarity with respect to attributes $A$ and $E$ is comparable. However, it is different for attribute $V$. Hence, numerical similarity values with respect to attribute $A$ or $E$ do not necessarily have the same meaning as an equal numerical similarity value with respect to attribute $V$. To illustrate this point, consider the case pair $\mathrm{C}_{10}$ and $\mathrm{C}_{23}$. In figure 1 , we can see that the similarity between $\mathrm{C}_{10}$ and $\mathrm{C}_{23}$ with respect to the volume $V$ is ' 0.72 ', which in linguistic terms can be considered as an 'average' volume similarity. However, if the similarity between $\mathrm{C}_{10}$ and $\mathrm{C}_{23}$ with respect to attribute angle was ' 0.72 ', we observe that this value is considered as a relatively low angle similarity compared to all other similarity values for that attribute and we conclude that the two cases are not very similar with respect to the attribute angle. That is, the same numerical similarity value has different meanings for different attributes. Thus, we can not compare directly the numerical values of the similarity and a simple sum of individual attribute similarities does not necessarily give an accurate representation of the similarity between two cases. In order to sensibly compare attribute similarities and generate an aggregate similarity measure, the CBR system has to understand what numerical similarity value actually constitutes a "high", "average" or "low" similarity for each attribute. We therefore propose the use of fuzzy sets. We define the fuzzy sets High, Avg and Low, which denote low similarity, average similarity and high similarity, respectively, for each attribute. The fuzzy membership functions of the three sets are defined for each attribute based on the minimum, maximum and average of the corresponding similarity values found across the case base. They, therefore, give a realistic indication of what constitutes a relatively "high similarity", "average similarity" or "low similarity" for an attribute.

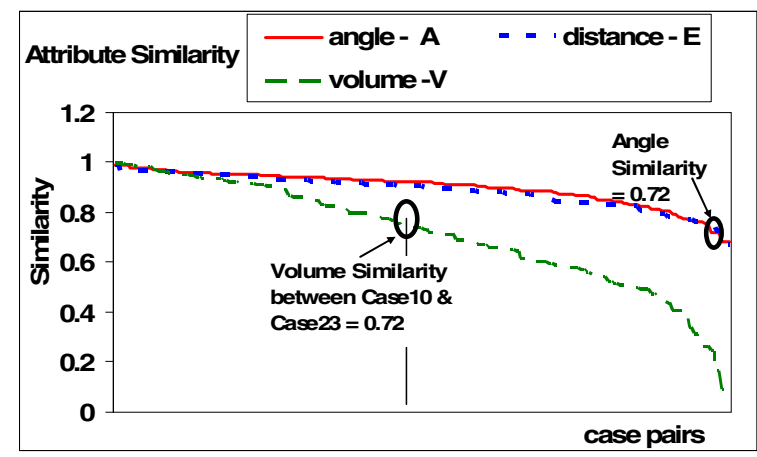

Fig. 1. The graph shows the similarity values obtained between all possible pairs of cases 
Figure 2 presents the fuzzy membership functions defined for attributes $A, E$ and $V$. The membership degree $\mu_{l, s}$ of similarity $s_{l}$ with respect to attribute $l$ is computed for each fuzzy set $s, H i g h, A v g$ and Low. The aggregate similarity consists of the High, Avg and Low component $\mathrm{M}_{s}$ defined as the sum of the membership degrees of the attribute similarities to the fuzzy sets High, Avg and Low of the corresponding attributes, shown in expression 3 .

$$
\mathbf{M}_{s}=\sum_{l=A, E, V}^{3} w_{l} \mu_{l, s} .
$$

where $w_{l}$ denotes the weight of attribute $l, l=A, E, V$ and $\mu_{1, s}, s=H i g h$, Avg, Low, is the membership degree of the attribute similarity to the fuzzy sets High, Avg and Low. A large value of component $\mathbf{M}_{\text {High }}$ indicates a high aggregate similarity between two cases, a large value of component $\mathbf{M}_{A v g}$ indicates an average similarity between two cases, while a large value of component $\mathbf{M}_{\text {Low }}$ indicates a low similarity between two cases. That is, $\mathbf{M}_{\text {High }}$ displays a net positive effect while $\mathbf{M}_{A v g}$ and $\mathbf{M}_{\text {Low }}$ display a net negative effect on the aggregate similarity. The aggregate similarity $s_{T}$ between two cases is defined as the net contribution $\mathbf{M}_{H i g h}, \mathbf{M}_{A v g}$ and $\mathbf{M}_{\text {Low }}$ as shown in expression 4.

$$
s_{T}=w_{H i g h} M_{H i g h}-w_{A v g} M_{A v g}-w_{\text {Low }} M_{\text {Low }} .
$$

The weights $w_{H i g h}, w_{A v g}$ and $w_{\text {Low }}$ determine the importance of the fuzzy sets High, Avg and Low respectively.
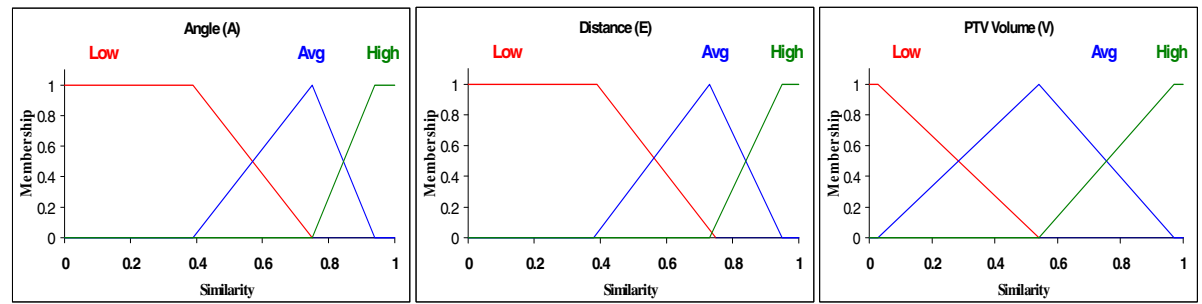

Fig. 2. Fuzzy membership functions for sets High, Low and Avg

\subsection{Non-linear Similarity}

Preliminary experiments have shown that the influence of the Angle similarity $\left(s_{A}\right)$ on the aggregate similarity is not linear. That is, if $s_{A}$ is high, then it is very likely that the treatment plan of the retrieved case is very suitable and contains a beam configuration that would achieve the desired radiation dose distribution for the target patient. However, if $s_{A}$ is low, then the corresponding treatment plan is very likely to be rather unsuitable for the target case. In other words, to retrieve cases whose treatment plans 
are very suitable for the target case, the aggregate similarity between cases has to be increased if the value $s_{A}$ is high and reduced or penalised if $s_{A}$ is low. This effect is not covered by the standard method of multiplying weights with the similarity value assigned to attributes, since a high weight for attribute $A$, would increase the aggregate similarity value not only if $s_{A}$ is high but also if $s_{A}$ is low.

For the similarity with respect to $V, s_{V}$, the effect is non-linear too, but in an opposite fashion. If $s_{V}$ is high, it does not substantially increase the likelihood that the treatment plan of the retrieved case is suitable. Similarly, if $s_{V}$ is low, it does not necessarily mean that the corresponding treatment plan is highly unsuitable i.e. the influence of both high and low similarity due to attribute $V$ is reduced or smoothed.

Expression 5 presents the aggregate similarity measure, which takes into account the non-linear effect of attributes, where $k_{l}$ is the non-linearity factor of attribute $l$.

$$
s_{T N L}=w_{H i g h} \sum_{l=A, E, V}^{3} w_{l}\left(\mu_{l, H i g h}\right)^{k_{l}}-w_{A v g} \sum_{l=A, E, V}^{3} w_{l}\left(\mu_{l, A v g}\right)^{k_{l}}-w_{L o w} \sum_{l=A, E, V}^{3} w_{l}\left(\mu_{l, L o w}\right)^{k_{l}}
$$

To achieve a stretching non-linear effect, $k$ should be smaller than 1 , to achieve a smoothing non-linear effect, $k$ should be greater than 1 and $\mathrm{k}$ should be equal to 1 if no non-linear effect is desired. After initial experiments, we have used $k=1 / 3$, for attribute $A ; k=3$, for attribute $V$; and $k=1$, for attribute $E$.

\section{Experimental Results}

We tested the performance of the fuzzy non-linear similarity measure using brain cancer patient cases. Since all cases contain the treatment plan used in that case, we can evaluate the similarity measure by comparing the treatment plan of the retrieved case with the actual treatment plan. In the experiments, we looked mainly at the beam configuration of the plan, in particular the beam angles. The beam angles consider the position of the gantry, the patient couch and the beams. The difference in the beam angle values in the plan of the retrieved case and the actual treatment plan of the target case gives the error. The error is averaged over all cases to give an indication of the quality of the similarity measure. A commonly employed evaluation strategy in CBR is the leave-one out cross validation method. Each case in the case base is consecutively made the target case and the most similar case among the remaining cases in the case base is retrieved. In the future when more cases are available, the case base can also be divided into training and test cases. Figure 3 a shows the average errors in angle degrees per beam across all cases obtained when using the weighted nearest neighbour similarity measure $(\mathrm{wNN})$, the fuzzy similarity measure (wFuzzy) given in expression (4) and the fuzzy non-linear similarity measure (wNL) given in expression (5). Following discussion with hospital staff, attribute $A$ is the most important, followed by $E$ and $V$. Therefore, the attribute weights are set at $\mathrm{w}_{\mathrm{A}}=0.6$, $w_{E}=0.3$ and $w_{\mathrm{V}}=0.1$ for all similarity measures. The weights for the fuzzy sets High, Low and Avg are set at $w H i g h=3, w L o w=2$ and $w A v g=1$. 

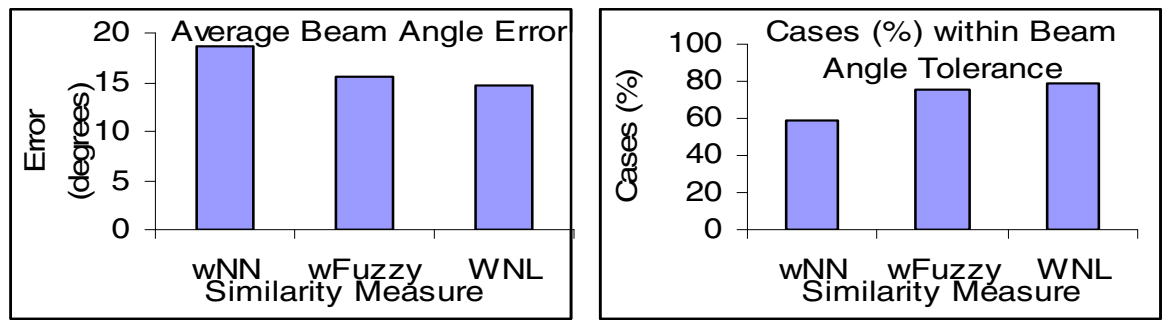

Fig. 3. a) The average error between the beam angles of the treatment plan of the retrieved case and the actual treatment plan. b) The percentage of cases, for which the similarity measure retrieves a treatment plan with acceptable beam angle difference.

We can see that the fuzzy similarity measure, wFuzzy, has a lower average error $\left(18.6^{\circ}\right)$ than the nearest neighbour method, wNN $\left(15.5^{\circ}\right)$. The error is further reduced with the fuzzy non-linear similarity measure, wNL $\left(14.7^{\circ}\right)$. Following consultation with hospital staff, an angle error of $20^{\circ}$ per beam is acceptable for most cases. Figure $3 \mathrm{~b}$, shows the number of cases for which the similarity measure retrieves a case, in which the average error per beam is less than $20^{\circ}$. We can see that the percentage of cases within this beam angle tolerance is $58 \%$ for $w N N, 75 \%$ for wFuzzy and $79 \%$ for wNL similarity measure.

\section{Conclusion}

In this paper, we described a case-based reasoning system (CBR) that is under development to aid oncologists with radiotherapy treatment planning in brain cancer.

The results confirm the assumption that similar treatment plans can be derived from similar geometric patient descriptors, as long as these descriptors are combined in an appropriate fashion in the similarity measure. Therefore, a key characteristic of our system is the fuzzy non-linear similarity measure that computes the similarity between cases in order to retrieve the case with the most suitable treatment plan. The fuzzy nature of the similarity measure allows aggregation of the similarity values with respect to single attributes to provide an accurate representation of the total similarity while taking into account the non-linear behaviour of attributes. The method was compared to the commonly used nearest neighbour method. The experimental results using real brain cancer patient cases show an improvement with the fuzzy non-linear similarity measure. The attribute weights and the non-linearity factor $k$ have been set empirically, but the performance of the similarity measure can be improved by optimising the weights. A detailed weight analysis to identify the optimum values of the attribute weights and $k$ is currently in process. We are aiming to find a set of weights that return the lowest average error with the leave-one-out cross validation method. Preliminary results show a significant reduction in beam angle error with optimized attribute weights.

In CBR systems the performance of the case retrieval mechanism and its similarity measure is crucial to the working of a CBR system. However, frequently, differences do exist between the target case and the retrieved case. Once the most similar case has 
been retrieved, its solution generally has to be adapted to the specific needs of the target case. Adaptation can be done by adjusting the beam configuration according to the geometric displacement in the location of the tumour and OAR structures of the target case compared to the retrieved case. Another method evaluates the plan using a treatment planning system to identify dose violations of OAR and confirm tumour coverage. The adaptation module of the CBR system then has to tweak the plan parameters of the retrieved case to resolve the violations. Adaptation can be carried out using if-then rules or by using another case-based reasoning inference mechanism.

Acknowledgements. The authors would like to thank the Engineering and Physics Science Research Council (EPSRC), UK, (Ref No. EP/C549511/1) and the City Hospital, Nottingham University Hospitals NHS Trust (UK) for their support.

\section{References}

1. Kolodner, J.: Case-Based Reasoning. Morgan Kaufmann, San Francisco (1993)

2. Oldham, M., Khoo, V.S., Rowbottom, C., Bedford, J.L., Webb, S.: A Case Study Comparing the Relative Benefit of Optimizing Beam Weights, Wedge Angles, Beam Orientations and Tomotherapy in Stereotactic Radiotherapy of the Brain. Phys. Med. Biol. 43, 2123-2146 (1998)

3. Haas, O.C.L., Bumham, K.J., Mills, J.A.: Hybrid Optimisation Technique for Radiotherapy Treatment Planning. In: Proceedings of the 1998 IEEE International Conference on Control Application, Trieste, Italy (1998)

4. Holt, A., Bichindaritz, I., Schmidt, R., Perner, P.: Medical Applications in Case-Based Reasoning. The Knowledge Engineering Review 20(3), 289-292 (2006)

5. Schmidt, R., Montani, S., Bellazzi, R., Portinale, L., Gierl, L.: Cased-Based Reasoning for Medical Knowledge-Based Systems. International Journal of Medical Informatics 64, 355367 (2001)

6. Song, X., Petrovic, S., Sundar, S.: A Case-Based Reasoning Approach to Dose Planning in Radiotherapy. In: Wilson, D. (ed.) ICCBR 2007. LNCS (LNAI), vol. 4626, pp. 348-357. Springer, Heidelberg (2007)

7. Berger, J.: Roentgen: Radiation Therapy and Case-Based Reasoning. In: Proceedings of the Tenth Conference on Artificial Intelligence for Applications, pp. 171-177 (1994)

8. Cover, T.M., Hart, P.E.: Nearest Neighbor Pattern Classification. IEEE Transactions on Information Theory 13(1), 21-27 (1967)

9. Wang, R., Zeng, Y.: Nonlinear Nearest-Neighbour Matching and its Application in Legal Precedent Retrieval. In: Information Technology and Applications, ICITA 2005, vol. 1, pp. 341-346 (July 2005)

10. Cheng, C.B.: A Fuzzy Inference System for Similarity Assessment in Case-Based Reasoning Systems: An Application to Product Design. Mathematical and Computer Modelling 38, 385-394 (2003)

11. N.E.M.A.: DICOM - Digital Imaging and Communications in Medicine, http: / / Medical. Nema. Org/

12. Mishra, N., Petrovic, S., Sundar, S.: A Knowledge-light Nonlinear Case-based Reasoning Approach to Radiotherapy Planning. In: Proceedings of the 21st International Conference on Tools with Artificial Intelligence, ICTAI, pp. 776-783 (2009) 